\title{
Extracorporeal Membrane Oxygenation in Nivolumab Associated Pneumonitis
}

\author{
Thomas-Michael Schneider ${ }^{1 *}$, Friederike Klenner ${ }^{2}$, Franz Brettner ${ }^{1}$ \\ 1 Department of Intensive Care Medicine, Krankenhaus Barmherzige Brueder, Munich, Germany \\ 2 Department of Internal Medicine V, University of Munich, Germany
}

\begin{abstract}
Background: Newly approved immunotherapeutic agents, like CTLA-4 inhibitors and antibodies against PD-1, are a promising therapeutic option in cancer therapy.

Case presentation: A 74-year-old man, with a history of advanced stage melanoma and treatment with ipilimumab, pembrolizumab and nivolumab, was admitted to the hospital due to respiratory failure with hypoxemia and dyspnoea. He rapidly developed severe acute respiratory distress syndrome (ARDS), which required treatment in the intensive care unit which included mechanical ventilation and extracorporeal membrane oxygenation (ECMO). Computed tomographic imaging (CT) showed signs of a pneumonitis, with an ARDS pattern related to the use of PD-1 antibodies. Treating the patient with high-dose immunosuppressive steroids led to an overall improvement. He was transferred to a rehabilitation hospital and subsequently to his home.

Discussion and conclusion: This is a unique case report of a patient suffering a grade 4 adverse event under nivolumab who survived having been treated with ECMO. It highlights the possibility of associated adverse reactions as well as the use of ECMO in palliative care patients. ECMO can be of great success even in patients with malignancies, but careful decision making should be done on a case by case basis.
\end{abstract}

Keywords: Nivolumab, pneumonitis, adult respiratory distress syndrome, extracorporeal membrane oxygenation, melanoma

Received: 27 February 2017 / Accepted: 21 April 2017

\section{INTRODUCTION}

Recently developed immunotherapeutic agents, such as check point inhibitors and antibodies against programmed cell death 1 (PD-1), open up new horizons in cancer therapy. The PD-1 antibody nivolumab has been approved for the treatment of malignant melanoma and lung cancer by the European Medicines Agency (EMA) since 2015.

Serious adverse events are comparably rare [1]. Today the use of extracorporeal membrane oxygenation (ECMO) is a worldwide established method for treating severe lung failure [2]. We report the successful use of extracorporeal membrane oxygenation (ECMO) in pneumonitis with ARDS-pattern associated with the use of PD-1 antibody nivolumab.

\section{CASE PRESENTATION}

A 74-year-old man with a body mass index (BMI) of $27.7 \mathrm{~kg} / \mathrm{m}^{2}$ ), was admitted to the emergency depart- ment of the Barmherzige Brueder Hospital in Munich, Germany. He presented with acute dyspnoea, muscle weakness, tiredness but no fever. Prehospital treatment with antibiotics had not resulted in any improvement in his condition. His medical history showed he had had a deep vein thrombosis and a pulmonary embolism one year previously. Two years earlier he had been diagnosed with a stage IV melanoma. He had received immunotherapy, first with ipilimumab and sequentially with pembrolizumab. Due to the advancement of his condition he received nivolumab, $3 \mathrm{mg} / \mathrm{kg}$ every two weeks for six cycles. The last cycle was received thirteen days before admission to the hospital.

Clinical findings: Breathing rate was $23 / \mathrm{min}, \mathrm{SpO}_{2}$ $76 \%, \mathrm{FiO}_{2}$ 0.21, heartrate $97 \mathrm{bpm}$, blood pressure $150 / 80 \mathrm{mmHg}$, and temperature $36.8^{\circ} \mathrm{C}$. Blood gas: $\mathrm{SaO}_{2} 92 \%$, with $8 \mathrm{l} / \mathrm{min}$ supplementary oxygen, $\mathrm{pO}_{2} 64$ $\mathrm{mmHg}, \mathrm{pCO}_{2} 24 \mathrm{mmHg}$, lactate $4.7 \mathrm{mmol} / \mathrm{l}, \mathrm{pH} 7.40$ and $\mathrm{BE}-8.2 \mathrm{mmol} / \mathrm{l}$.

Correspondence to: Thomas-Michael Schneider, Abteilung für Intensivmedizin, Krankenhaus Barmherzige Brueder Muenchen, Romanstr. 93, 80639 Muenchen, Germany. E-mail: tms@live.de 

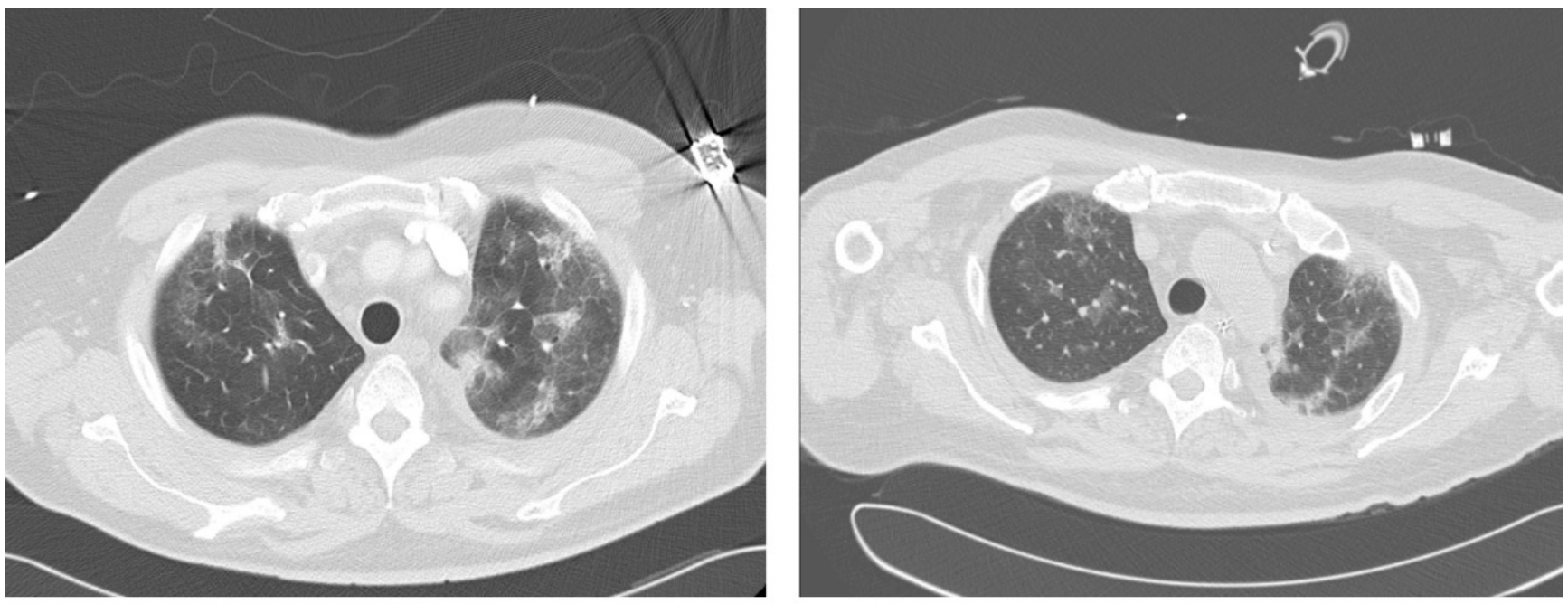

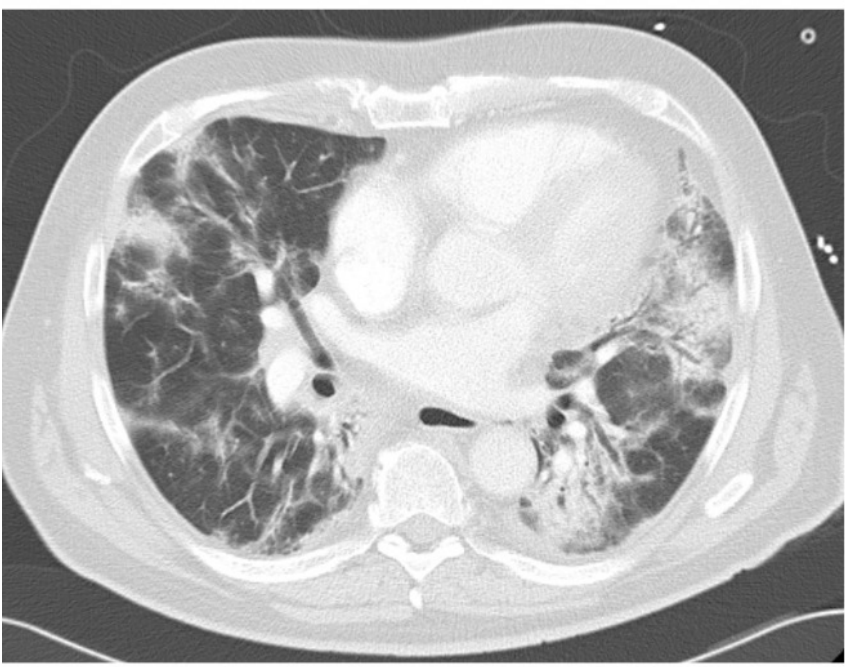

Day 1

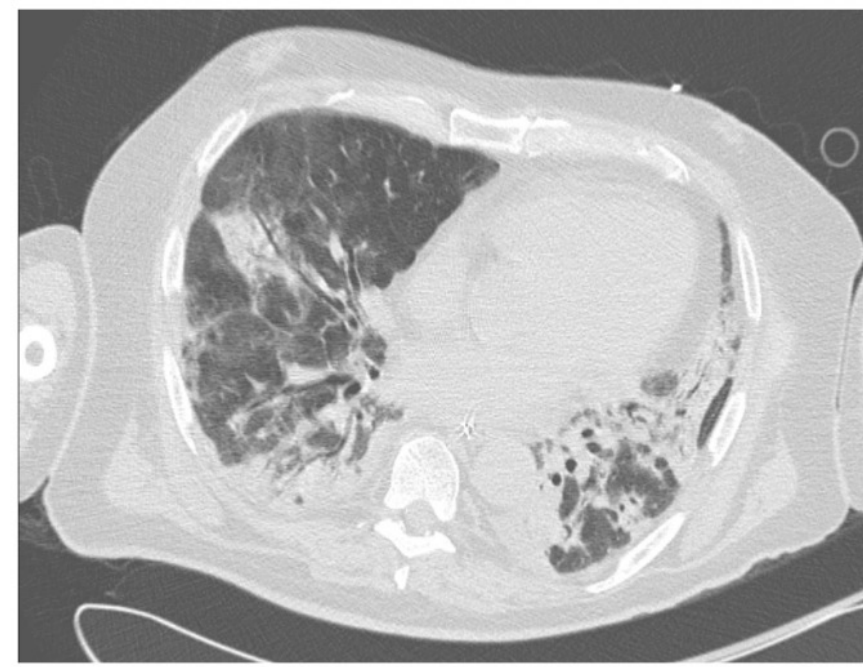

Day 17

Fig. 1. CT-Scan, with contrast agent, on admission (left side) and high-resolution computer tomography during treatment (right side).

Relevant laboratory results: Leukocytes $15.4 \times 10^{3} / \mu \mathrm{l}$, CRP 76mg/l, troponin T 0.0 7ng/ml, D-dimer $4052 \mu \mathrm{g} / \mathrm{l}$, ALAT $232 \mathrm{U} / \mathrm{l}$, creatinine $1.85 \mathrm{mg} / \mathrm{dl}$, GFR $38 \mathrm{ml} / \mathrm{min}$.

An initial differential diagnosis of a severe community-acquired pneumonia or an acute pulmonary embolism was considered. The echocardiography showed a Grade 1 mitral valve insufficiency, and diastolic dysfunction. There was no evidence from the CT of a pulmonary embolism, but diffuse bilateral infiltrations with consolidations and enlarged mediastinal lymph nodes were seen (Figure 1).

The therapeutic interventions and course is presented in Figure 2.

After collecting blood cultures, calculated empiric antibiotic therapy with piperacillin-tazobactam $(4.5 \mathrm{~g}$, $3 \mathrm{x}$ per day) and clarithromycin (500mg $2 \mathrm{x}$ per day) was initiated. The patient was transferred to the inten- sive care unit (ICU) in a stable cardio-circulatory condition, but with a serious respiratory failure and the need for immediate non-invasive ventilation. Twenty-four hours after his admission to the hospital, the patient's condition deteriorated due to exhaustion and intubation with mechanical ventilation was required. He quickly developed a severe ARDS, with a recorded oxygenation index of $75 \mathrm{mmHg}$, compliance of 18 $\mathrm{ml} / 100 \mathrm{~Pa}$, and a peak inspiratory pressure of $4 \mathrm{kPa}$.

These conditions led to the decision to initiate a venovenous extracorporeal membrane oxygenation using an ILA-activve ${ }^{\circledast}$ system (Novalung, Heilbronn, Germany). Cannulas were placed in the right femoral vein (French 23) and the right internal jugular vein (French 19). The ECMO circuit was set at blood flow $41 /$ minutes, and oxygen flow $91 /$ minutes and this allowed the mechanical ventilation to be reduced to an ultra protective level. 

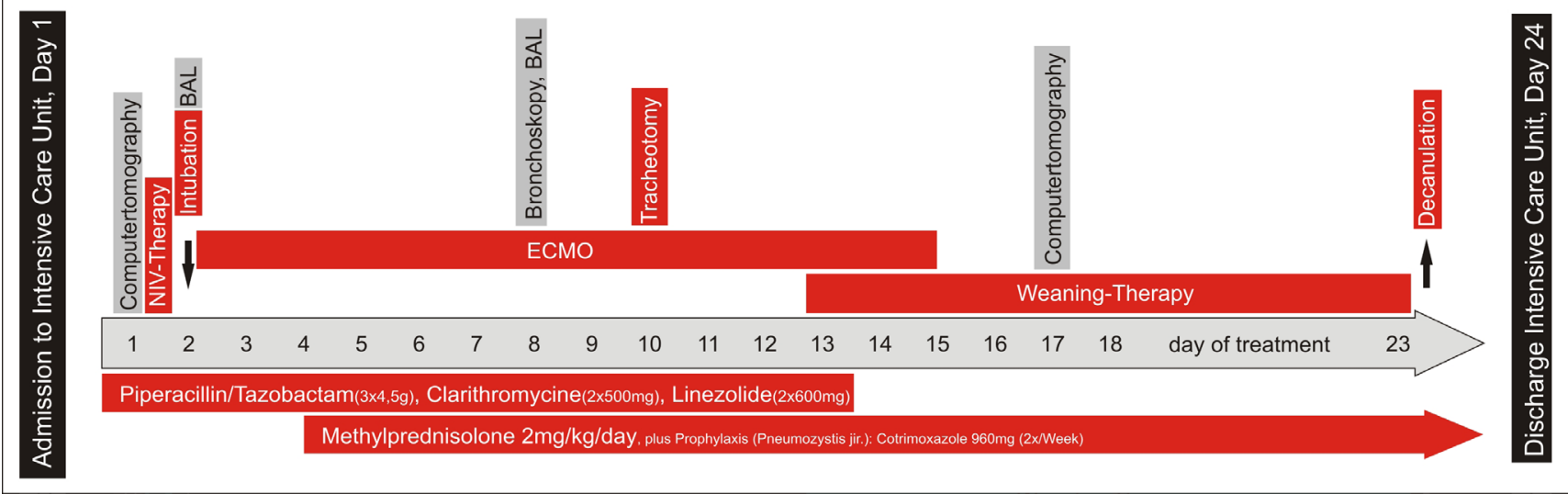

Fig. 2. Diagnostic and therapeutic interventions during treatment.

BAL: bronchoalveolar lavage, ECMO: extracorporeal membrane oxygenation, NIV: non-invasive ventilation.

Due to possible MRSA colonisation, the antibiotic regimen was intensified with the addition of linezolide, $600 \mathrm{mg}$ every 12 hours by intravenous infusion over 30-120 minutes.

Haemodynamic monitoring was performed with $\mathrm{PiCCO}^{\circledR}$ (Pulsion Medical Systems SE, Feldkirchen, Germany). Initially, the infectious parameters slightly increased but rapidly decreased over the following few days of therapy. (Figure 3). There was no bacterial, fungal or viral growth from repeated broncho-alveolar lavage or blood cultures. The patient's temperature was never recorded as being higher than $38.5^{\circ} \mathrm{C}$.

Considering the patient had nivolumab induced pneumonitis, it was decided, after four days of mechanical ventilation, to start an immunosuppressive therapy with methylprednisolone, $2 \mathrm{mg} / \mathrm{kg} /$ day together with pneumocystis pneumonia prophylaxis with cotrimoxazole, 960mg twice a week. Nine days after intubation, a dilative tracheostomy was performed. After thirteen days, the pulmonary gas exchange had improved to the extent that it was possible to end ECMO therapy. Complications associated with ECMO therapy did not occur. At day seventeen, a high-resolution CT showed a slight improvement in the pulmonary infiltration though there remained a diffuse bilateral ground glass opacity with consolidations in the lower lobes and bronchial thickening. (Figure 1)

During the following weaning period, prolonged wake-up trials and daily physiotherapy were given resulting in the oxygen supply being reduced. Eight days later the patient was in a good cognitive and cardiopulmonary condition, and one day before he was discharged from the ICU, the tracheostomy tube was removed. After three weeks of rehabilitation, he went home, without impairments besides a slight weakness. He gave written consent in publishing this case.

\section{DISCUSSION}

The promising ratio between benefit and adverse events, as well as a widening range of indications, are likely to result in an increasing use of new immunotherapeutic agents like ipilimumab, nivolumab or pembrolizumab. However, it is perceived that a growing number of people will report with moderate or severe adverse events related to these drugs. In a recent study [3], $50 \%$ of the patients treated with the CTLA-4 inhibitor, ipilimum$\mathrm{ab}$, experienced Grade 3 adverse events requiring admission to a hospital or Grade 4 life threatening events. Severe adverse reactions with nivolumab seem to occur less often [4]. The data suggests a better therapeutic outcome when sequential therapy with ipilimumab followed by nivolumab is prescribed for the treatment of malignant melanoma [5]. The incidence of nivolumab associated pneumonitis is $2.2-6 \%$, and pneumonitis associated mortality is $0.7 \%$ [6].

There are positive reports to treat Grade 3 and 4 adverse events with $2-4 \mathrm{mg} / \mathrm{kg}$ prednisolone. If there is no response, another immunosuppressant such as infliximab can be administered $[7,8]$.

The use of ECMO is now an established method for the treatment of severe lung failure. In the present case, ECMO was used to allow the prednisolone therapy to improve lung function and to allow an ultra-protective mechanical ventilation level and the avoidance of further ventilator-induced lung injury [9]. Jugular or femoral veins large enough to ensure a sufficient blood flow are essential, as is a team experienced in ECMO therapy 


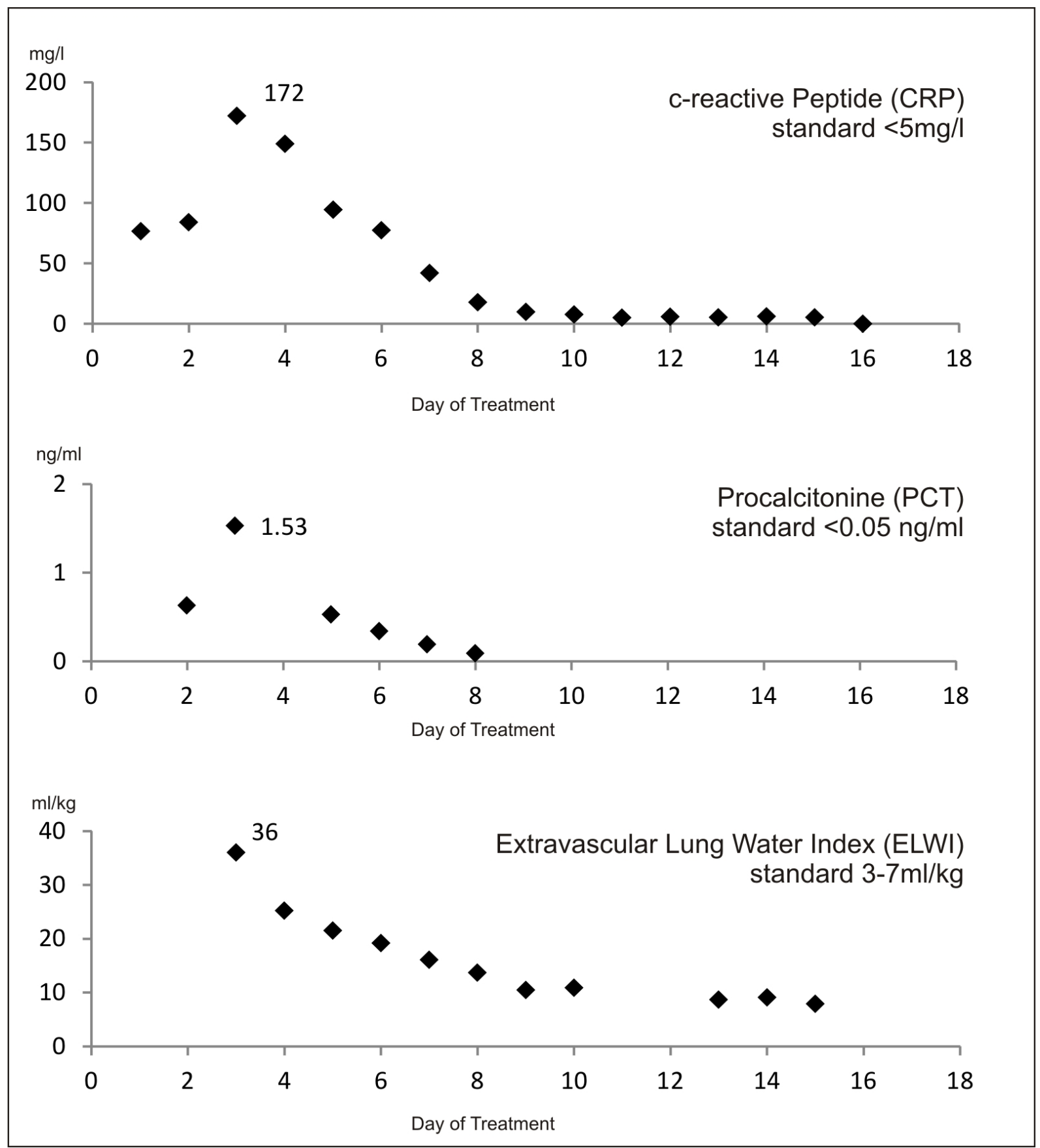

Fig. 3. Important parameters in the course of treatment: C-reactive peptide (CRP), procalcitonin (PCT), extravascular Lung water index (ELWI)

and possible complications. Common complications are haemolysis, haemorrhage, infection, pump failure and dislocation of an ECMO-cannula. Those complications occur much more frequently in veno-arterial than in veno-venous ECMO therapy [2], and for this reason, the latter can be considered somewhat safer. Also, it is superior in treating an isolated lung failure compared to veno-arterial-ECMO, because pre-oxygenated blood in the pulmonary artery reduces the right ventricular afterload caused by hypoxic pulmonary vasoconstriction. Furthermore, selective hypoxemia of the coronary arteries and the cerebral vessels cannot occur.

ECMO therapy in patients with malignancy is associated with a higher mortality rate, compared to patients without malignancy [10] due to the underlying disease and inherent complications. ECMO therapy should not be considered if the patient's health status is already impaired and improvement or maintenance of quality-of- 
life seems unlikely. Taking into account that the risks of serious complications under veno-venous ECMO are less frequent, the decision whether to perform ECMO or not can be more liberal when dealing with an isolated lung failure. In our case, the patient presented with a one organ failure but his general health was otherwise good. The patient retrospectively confirmed that he was grateful for the decision to use ECMO.

\section{CONCLUSION}

It is our opinion that, in general, patients with malignancies should not be excluded from ECMO therapy. Decisions, established on a case by case basis and considering all benefits and risks, should clarify which patients are likely to benefit from ECMO.

It should be borne in mind that the newer immunotherapeutic agents can cause life-threatening pneumonitis, but when identified promptly, can be successfully treated.

\section{ACKNOWLEDGMENT}

CT-Scans by courtesy of the Department of Diagnostic and Interventional Radiology, Krankenhaus Barmherzige Brueder, Munich

\section{CONFLICT OF INTEREST}

All authors declare that there are no conflicts of interest.

\section{REFERENCES}

1. Kobold S DP, Schnurr M, Subklewe M, RothenfusserS, Endres S. Immunotherapy in tumor-activated T-cells as a new treatment modality. Dtsch Arztebl Int. 2015;112:809-15.

2. Makdisi G, Wang IW. Extra Corporeal Membrane Oxygenation (ECMO) review of a lifesaving technology. J Thorac Dis. 2015;7:E166-76.

3. Hodi FS, O'Day SJ, McDermott DF, et al. Improved survival with ipilimumab in patients with metastatic melanoma. N Engl J Med. 2010;363:711-23.

4. Robert C, Long GV, Brady B, et al. Nivolumab in previously untreated melanoma without BRAF mutation. N Engl J Med. 2015;372:320-30.

5. Postow MA, Chesney J, Pavlick AC, et al. Nivolumab and ipilimumab versus ipilimumab in untreated melanoma. N Engl J Med. 2015;372:2006-17.

6. Squibb B-M. Opdivo (Nivolumab), Immune-Mediated Adverse Reactions Management Guide. (packet insert) 2015.

7. Villadolid J, Amin A. Immune checkpoint inhibitors in clinical practice: update on management of immune-related toxicities. Transl Lung Cancer Res. 2015;4:560-75.

8. Nishino M, Sholl LM, Hodi FS, Hatabu H, Ramaiya NH. Anti-PD1-Related Pneumonitis during Cancer Immunotherapy. N Engl J Med. 2015;373:288-90.

9. Amato MB, Barbas CS, Medeiros DM, et al. Effect of a protectiveventilation strategy on mortality in the acute respiratory distress syndrome. N Engl J Med. 1998;338:347-54.

10. Wohlfarth P, Ullrich R, Staudinger T, et al. Extracorporeal membrane oxygenation in adult patients with hematologic malignancies and severe acute respiratory failure. Critical care. 2014;18:R20. 